\title{
Effect of Heat Treatment on Optical Characteristics of Highly Nonlinear Optical Fiber Doped with PbTe Semiconductor Quantum Dots
}

\author{
Seongmin Ju, Pramod R. Watekar, and Won-Taek Han \\ Department of Information and Communications, Gwangju Institute of Science and Technology \\ 1 Oryong-dong, Buk-gu, Gwangju, 500-712, South Korea \\ Tel: 82-62-970-2215, Fax: 82-62-970-2204,E-mail: wthan@gist.ac.kr \\ Chuljin Kim \\ Department of Ceramic Engineering, Gyeongsang National University \\ 900 Gajwa-dong, Jinju, Gyeongnam, 660-701, South Korea
}

\begin{abstract}
The effect of heat treatment of highly nonlinear optical fiber doped with the PbTe nanoparticles on absorption characteristics was investigated. The excitonic absorption peaks near 687 and $1055 \mathrm{~nm}$ were found linearly red-shifted with temperature.

(C) 2005 Optical Society of America

OCIS codes: (000.6850) heat treatment; (060.2400) nonlinearity; (060.2310) optical fiber; (000.2170) image furnace
\end{abstract}

Glasses doped with semiconductor nano-particles are known to have a large optical nonlinearity and hence, they are of great interest for all-optical devices applications [1]. Especially, IV-VI semiconductor quantum dots (SQDs) of lead chalcogenides such as $\mathrm{PbS}, \mathrm{PbSe}$, and $\mathrm{PbTe}$ are candidates for optical communication applications because of their narrow band-gap, large nonlinearity, and fast response time [2]. Recently, we have developed, for the first time to the best of our knowledge, the fibers doped with PbTe nano-particles and the unique features of the fibers and the high nonlinear refractive index have drawn much attention [3-5].

The absorption peaks in the PbTe SQDs doped optical fiber arise due to excitonic resonance from the SQD. Thus the peak position of the optical absorption due to PbTe SQDs is a key for high nonlinear optical effect. The absorption peaks can be shifted towards longer wavelength by increasing the size of the precipitated PbTe SQDs. It is known to increase the size of compound semiconductor particles in glass matrix by diffusion induced growth mechanism through a proper heat treatment.

In this paper, we proposed a new method to heat treat an optical fiber. It is practically impossible to heat treat a bare fiber in a conventional box furnace because of fragility of the fiber after heat treatment. The heat treatment of the optical fiber was carried out in the "image furnace", which uses halogen lamps as a heat source. The radiation of the halogen lamp passes through the optical fiber and thus, the PbTe SQDs in the core can be directly heat treated. This heat treatment by light radiation is advantageous because surface contamination as well as oxidation of the optical fiber is minimized. The effect of heat treatment on the absorption characteristics of the fiber was investigated and the growth of the PbTe SQDs versus heat treatment temperature was also discussed.

Optical fiber was manufactured using the MCVD process and the PbTe nano-particles were incorporated in the core using atomization technique [5]. The doping solution for the atomization process was prepared by dissolving reagent grade $\mathrm{PbO}$ and $\mathrm{Te}$ powders in nitric acid solution to form the $\mathrm{PbTe}$ concentration of $0.03 \mathrm{M}$. The core diameter and the cutoff wavelength of the PbTe doped fiber were $9.6 \mu \mathrm{m}$ and $1.19 \mu \mathrm{m}$, respectively.

Optical absorption spectrum of the optical fiber incorporated with $\mathrm{PbTe}$ nano-particles in the core was measured by cut-back method where we used the white light source (Ando AQ 4303B) for launching the input broadband light and the output spectrum was measured with OSA (Ando AQ 6315B). To investigate the effect of the heat treatment on the optical fiber, we heat treated the optical fiber at 700,1000 , and $1100{ }^{\circ} \mathrm{C}$ for an hour using the halogen lamp in the image furnace. Fig. 1 shows the structure of the image furnace. To focus the light on to a fiber in the image furnace, the shape of the mirror part was made by using a following elliptic equation.

$$
\frac{X^{2}}{a^{2}}+\frac{Y^{2}}{b^{2}}=1, \quad \frac{a}{b}=0.8
$$




\section{NFB4}

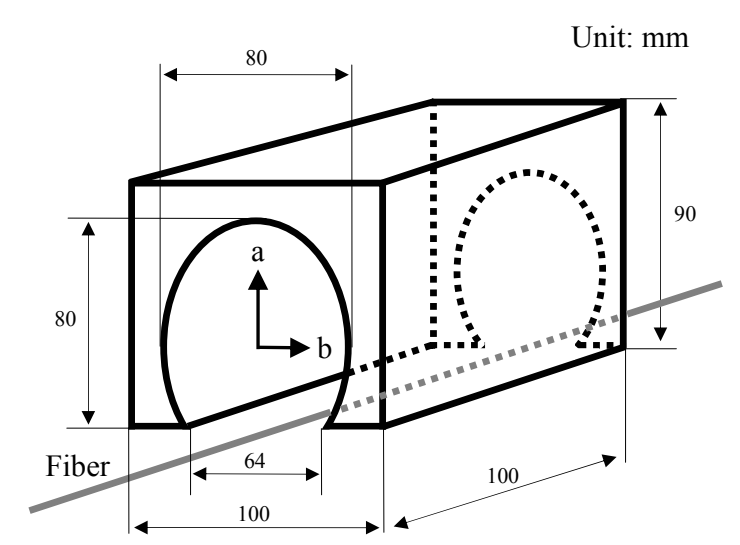

Fig. 1. Dimension of the mirror part in the image furnace using halogen lamps.

Fig. 2 shows the X-ray diffraction pattern of the PbTe-doped preform prepared by the MCVD and the atomization process. As a reference, XRD of a preform made without doping process is also shown in the figure. The fiber preform doped with PbTe clearly shows a strong diffraction peak at $2 \theta=21.6^{\circ}$, which is due to nano-sized crystalline PbTe particles. Note that for the preform without dopants, there is no diffraction peak but a diffused background. This XRD result clearly indicates the presence of the PbTe particles formed in the core of the preform. The rather broad feature of the diffraction peak of the preform doped with $\mathrm{PbTe}$ is due to the effect size of the $\mathrm{PbTe}$ nanoparticles.

The absorption spectra of the PbTe SQDs doped optical fiber that were heat treated at various temperatures are shown in Fig. 3. The peaks at 687 and $1055 \mathrm{~nm}$ of the fiber before heat treatment are attributed to absorption of the $\mathrm{PbTe}$ semiconductor quantum dots in the fiber core region [6]. It is interesting to note that the PbTe particles, which were embedded in the core of preform, were still preserved in the core of the fiber even after drawing at high temperature (above $2150^{\circ} \mathrm{C}$ ). The absorption peak at $1380 \mathrm{~nm}$ is due to the $\mathrm{OH}$ impurities.

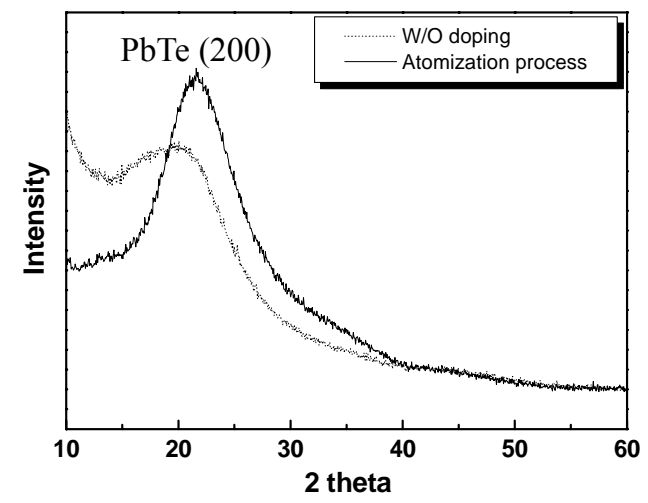

Fig. 2. XRD patterns of the PbTe-doped perform.

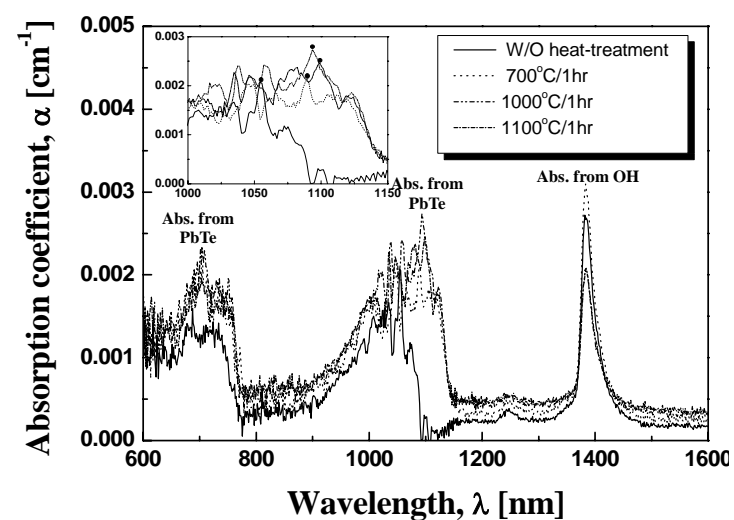

Fig. 3. Absorption spectra of PbTe-doped fiber by heat-treatment at 700,1000 , and $1100{ }^{\circ} \mathrm{C}$ for 1 hour. 


\section{NFB4}

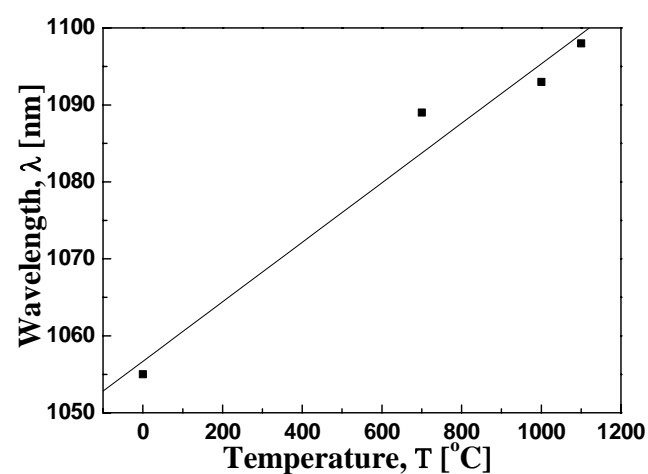

Fig. 4. Shift of the absorption peak at $1055 \mathrm{~nm}$ of the fiber doped with $\mathrm{PbTe}$ SQDs after heat-treatment at 700,1000 , and $1100^{\circ} \mathrm{C}$ for 1 hour.

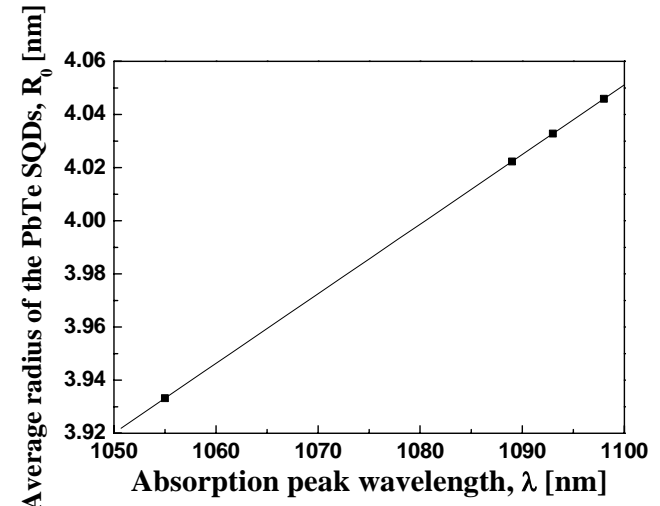

Fig. 5. Variation of average radius of the PbTe SQDs with absorption peak wavelength after heat-treatment at 700,1000 , and $1100^{\circ} \mathrm{C}$ for 1 hour.

As the temperature increased, the absorption peak wavelength around $1055 \mathrm{~nm}$ appeared to increase as shown in Figs. 3 and 4. The absorption peak shifted linearly towards longer wavelength with the increase of the heat treatment temperature. This red shift upon heat treatment can be explained in terms of the increase in the size of the PbTe SQDs. However, the extent of the peak shift was found to be different at different wavelengths. The peak shift of the absorption band at $1055 \mathrm{~nm}\left(43 \mathrm{~nm}\right.$ up to $\left.1100{ }^{\circ} \mathrm{C}\right)$ was larger than that at $687 \mathrm{~nm}\left(17 \mathrm{~nm}\right.$ up to $\left.1100{ }^{\circ} \mathrm{C}\right)$. Since the large sized PbTe SQDs have larger surface energy than the small ones, the growth rate of large particles became faster and thus the peak shift of the absorption band at longer wavelength became larger at the given heat treatment [7]. It is noted that the $\mathrm{OH}$ loss of the fiber decreased by the heat treatment, maybe due to dehydration at high temperatures during the heat treatment. The absorption at $1380 \mathrm{~nm}$ decreased when heat treatment temperature was $1000^{\circ} \mathrm{C}$ and $1100^{\circ} \mathrm{C}$, while there was no decrease in absorption at $700{ }^{\circ} \mathrm{C}$.

The size of the PbTe particles can be calculated using the following equation $[3,5]$.

$$
E-E_{b}=h^{2} /\left(8 \mu R_{0}^{2}\right)
$$

where $R_{0}$ is the mean radius of the $\mathrm{PbTe}, E$ is the energy for the first exaction peak, $E_{b}(337 \mathrm{meV})$ is the band gap energy for the bulk PbTe, $h$ is the Plank constant, and $\mu$ is the reduced mass determined by $1 / \mu=1 / \mu_{e}+1 / \mu_{h}$ with $\mu_{e}$ $=\mu_{h}=0.058 \mathrm{~m}_{\mathrm{e}}, \mathrm{m}_{\mathrm{e}}$ being the electron mass. Using the equation (2), the mean radius of PbTe particles was calculated to be $3.933,4.022,4.033$, and $4.046 \mathrm{~nm}$ before and after heat treatment at 700,1000 , and $1100{ }^{\circ} \mathrm{C}$, respectively as shown in Fig. 5. The total increase in the average radius of the precipitated PbTe particles was $0.113 \mathrm{~nm}$ after the heat treatment up to $1100^{\circ} \mathrm{C}$.

In summary, we investigated the effect of heat treatment on the absorption characteristics of the fiber doped with the nano-sized PbTe SQDs. Incorporation of the PbTe quantum dots in the fiber core was confirmed by the absorption peaks appeared at $687 \mathrm{~nm}$ and $1055 \mathrm{~nm}$ and their shift upon heat treatment. The absorption peak was found to shift linearly toward longer wavelength after heat treatment at 700,1000 , and $1100{ }^{\circ} \mathrm{C}$ for 1 hour. The red shift of the excitonic absorption peak was attributed to the increase in average size of the PbTe quantum dots in the fiber core. A new heat treatment method for optical fibers was also proposed by adopting so-called image furnace where contamination and oxidation of the fiber were minimized.

\section{References}

[1] U. Woggen, Optical Properties of Semiconductor Quantum Dots, (Springer-Verlag Berlin Heidelberg 1997).

[2] R. Dalven, "A Review of the Semiconductor Properties of PbTe, PbSe, PbS and PbO," Infrared Phy., 9, 141-184 (1969).

[3] J. S. Cho, U.-C. Peak, W.-T. Han, and J. Heo, "Fabrication and heat treatment effects on absorption characteristics of glass fibers doped with PbTe semiconductor quantum dots," OFC 2001, ThC4-1 ThC4-3 (2001).

[4] W.-T.Han and Y. H. Kim, "Linear and nonlinear optical properties of optical fibers containing PbTe quantum dots for all optical switching application," The 2nd International Korea-China Symposium on Glass-Ceramics, 34 40 (2002).

[5] S. Ju, Y. H. Kim, W.-T. Han, "Fabrication of PbTe Doped Optical Fiber by Using Atomization Doping Process and its Optical Nonlinearity," ECOC 2004 Proceedings-Vol. 4, Th2.3.3, 850 851 (2004).

[6] V. C. S. Reynoso, A. M. de Paula, R, F. Cuevas, J. A. Medeiros Neto, O. L. Alves, C. L. Cesar and L. C. Barbosa, "PbTe quantum dot doped glasses with absorption edge in the $1.5 \mu \mathrm{m}$ wavelength region," Electron. Lett., Vol. 32, 12, 1013 1014 (1995).

[7] Ng, J. D., Lorber, B., Witz, J., Theobald-Dietrich, A., "The crystallization of biological macromolecules from precipitates: Evidence for Ostwald ripening," J. Cryst. Growth 168, 50 (1996). 